\title{
Relationship between bronchial and arterial diameters in normal human lungs ${ }^{1}$
}

\author{
N BEREND, ANN J WOOLCOCK, AND G E MARLIN \\ From the Repatriation General Hospital, Concord, and the Department of Medicine, \\ University of Sydney, Sydney, Australia
}

ABSTRACT In order to find an objective method for measuring narrowing of small airways, eight lungs from four people without lung disease were inflated and fixed at $25 \mathrm{~cm}$ of water pressure. Eight to nine blocks were taken at random from each of six parasaggital slices from each lung, sectioned, and stained. The size of the bronchioles (airways without cartilage) in relation to their accompanying arteries was determined. The internal bronchiolar diameter was compared with three different arterial diameters (the internal, external medial, and external adventitial). The ratio of the internal bronchiolar diameter to the external adventitial arterial diameter was constant between lungs $(0.62 \pm 0.02)$ and independent of the method of inflation or the position within the lungs. This ratio may provide a useful index of bronchiolar narrowing in disease.

In recent years much interest has centred on the importance of pathological changes in small airways as a cause of the lung function changes recorded in chronic bronchitis and emphysema (Hogg et al, 1968; Martin et al, 1970; Macklem et al, 1971; Zamel et al, 1976). Small airways are defined in physiological terms as conducting airways with an internal diameter of less than $2 \mathrm{~mm}$, which includes bronchioles as well as some small bronchi. Several morphometric methods have been used to assess narrowing of small airways, but none of them has allowed the size of individual small airways to be assessed.

One approach has been to plot the size distribution of small airway diameters or cross-sectional areas and compare them with normal distributions (Bignon et al, 1970; Linhartová et al, 1971, 1973; Depierre et al, 1972; Matsuba and Thurlbeck, 1972). If an excess of airways of a particular size range is found with a deficit in a larger range presumably narrowing of the larger airways has occurred. Similarly, an overall reduction of the cross-sectional area of small airways has been interpreted as narrowing. Another approach has been to measure the ratio of the internal to external bronchial diameters (Martin et al, 1968, 1970). This ratio, however, is influenced both by changes in wall thickness and changes in luminal diameter.

${ }^{1}$ Supported by a grant from the Department of Veterans Affairs, Australia.
Linhartová et al (1977) were able to show bronchiolar deformities by comparing the crosssectional shape of the airways in lungs with emphysema with a mathematical model of the shape of normal airways cut at various planes.

We have studied the size relationship of bronchioles to their accompanying pulmonary arteries to determine if this could define predicted normal bronchiolar calibre and therefore serve to measure bronchiolar narrowing in disease.

\section{Materials and methods}

Four normal pairs of lungs were obtained at necropsy (patients A, B, C, and D). The sex, age, $>$ and causes of death of the patients are given in table 1. All were non-smokers with no past history of lung disease. Patient $C$ had suffered from ischaemic heart disease and had a history of congestive cardiac failure, but this was controlled at

Table 1 Description of patients by sex, age, and cause of death

\begin{tabular}{llll}
\hline Patient & Sex & Age & Cause of death \\
\hline A & M & 16 & Head injuries \\
B & F & 17 & Head injuries \\
C & M & 64 & Cerebrovascular accident \\
D & F & 60 & Carcinoma of the breast \\
\hline
\end{tabular}


the time of death. One of each pair of lungs was inflated by cannulating the main bronchus only (the right lungs of patients $A$ and $B$ and the left lungs of patients $C$ and $D$ ) and the other by cannulating both the main bronchus and the pulmonary artery (the left lungs of patients $A$ and $B$ and the right lungs of patients $\mathbf{C}$ and $\mathbf{D}$ ). Inflation was achieved with a $10 \%$ solution of formalin under a constant pressure of $25 \mathrm{~cm}$ of water for a period of 48 to 72 hours using a modification of the apparatus described by Heard (1958). The volume of the inflated lungs from patient $D$ were measured by water displacement. Parasaggital slices were obtained by cutting at $1 \mathrm{~cm}$ intervals starting at the lateral pleural surface. The slices were labelled one to seven in order from the lateral slice. Eight to ten blocks measuring $1.5 \times 2.5 \mathrm{~cm}$ each were taken at random from each slice using a template, except from slice three which was examined to exclude the presence of emphysema. The blocks from patient $D$ were further labelled according to whether they had been taken from the upper or lower halves of the lungs. Paraffin sections of $5 \mu \mathrm{m}$ thickness were prepared from the blocks and stained with haematoxylin and eosin. These were examined microscopically under 80 -power magnification with a measuring grid in the eyepiece. Airways free from cartilage and glands were located and their internal (IB) diameters measured. When a bronchiole was definitely accompanied by a pulmonary artery three arterial diameters, the internal (IA), external medial (EMA), and external adventitial (EAA) diameters were also measured (figs 1 and 2). The external adventitial diameter
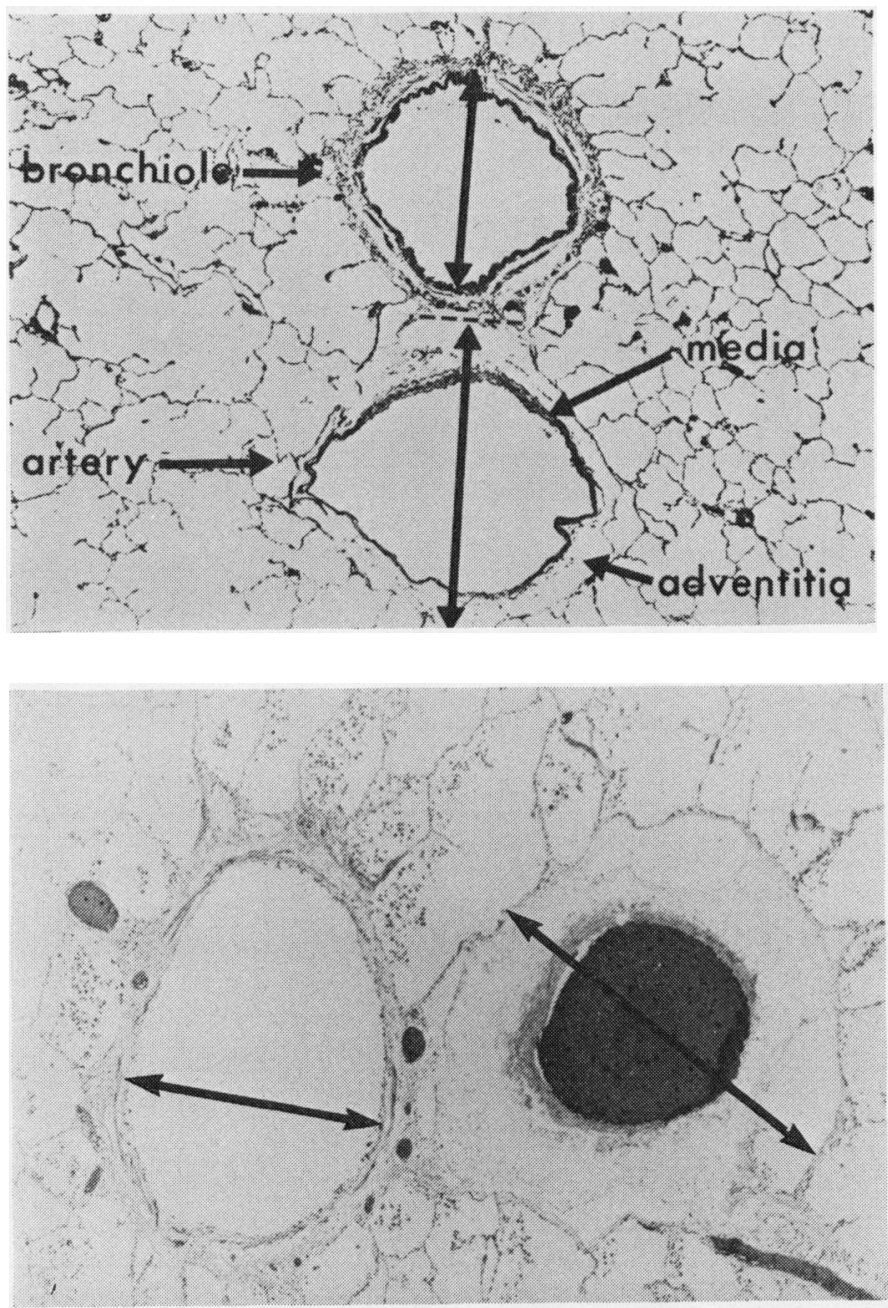

Fig 1 Pulmonary artery accompanying a bronchiole. Estimated junction is shown by dotted line. Internal bronchiolar and external adventitial arterial diameters are indicated by arrows.

Fig 2 Another example of a pulmonary artery accompanying a bronchiole. Arrows indicate measured diameters as in fig 1. 
was less well defined histologically than the other diameters. Only rarely, however, was real difficulty experienced in measuring it. When the bronchioles and arteries were contiguous (as in fig 1) the boundary between the two was estimated in the manner shown by the dotted line in the figure. When the arteries and bronchioles had been sectioned obliquely the lesser diameters were measured. Matsuba and Thurlbeck (1971) have shown previously that this method gives results approximating those obtained when only transversely cut airways are measured. A total of 1521 bronchioles and 1121 pairs of bronchioles and arteries were measured. The difference in numbers arises because not every bronchiole was accompanied by an artery. The results were expressed as the IB/IA, IB/EMA, and IB/EAA ratios for the individual pairs of structures.

Shrinkage during processing was presumably the same for bronchioles and arteries, and therefore no correction was made for shrinkage in determining the ratios. The diameter measurements, that is, the IB, IA, EMA, and EAA as well as the ratios, that is, the IB/IA, IB/EMA, and IB/EAA were analysed by a four-way analysis of variance to determine if systematic differences existed between individual patients, right and left lungs, methods of inflation, and individual slices. The data from the upper and lower halves of lungs (patient D only) were analysed by Student's $t$ test As the young and old patients were clustered around narrow age groups it was thought that statistical comparison of age-related differences was not justifiable.

\section{Results}

Table 2 shows the mean results and standard errors for all measurements and all ratios for the eight lungs, while table 3 shows the results of the

Table 2 All mean measured diameters ( $\mathrm{mm}$ ) and calculated ratios

\begin{tabular}{|c|c|c|c|c|c|c|c|}
\hline & $I B$ & $I A$ & $E M A$ & $E A A$ & $I B / I A$ & $I B / E M A$ & $I B / E A A$ \\
\hline A Left & $\begin{array}{c}0.45 \\
(0.02)\end{array}$ & $\begin{array}{c}0.34 \\
(0.02)\end{array}$ & $\begin{array}{c}0.43 \\
(0.02)\end{array}$ & $\begin{array}{c}0.81 \\
(0.04)\end{array}$ & $\begin{array}{c}1.69 \\
(0.07)\end{array}$ & $\begin{array}{c}1 \cdot 19 \\
(0.03)\end{array}$ & $\begin{array}{c}0.59 \\
(0.01)\end{array}$ \\
\hline A Right & $\begin{array}{c}0.44 \\
(0.02)\end{array}$ & $\begin{array}{c}0.23 \\
(0.01)\end{array}$ & $\begin{array}{c}0.32 \\
(0.02)\end{array}$ & $\begin{array}{c}0.78 \\
(0.03)\end{array}$ & $\begin{array}{c}2.44 \\
(0.11)\end{array}$ & $\begin{array}{c}1.57 \\
(0.05)\end{array}$ & $\begin{array}{c}0.61 \\
(0.01)\end{array}$ \\
\hline B Left & $\begin{array}{c}0.34 \\
(0.02)\end{array}$ & $\begin{array}{c}0.26 \\
(0.02)\end{array}$ & $\begin{array}{c}0.34 \\
(0.02)\end{array}$ & $\begin{array}{c}0.68 \\
(0.03)\end{array}$ & $\begin{array}{c}1.86 \\
(0.08)\end{array}$ & $\begin{array}{c}1.25 \\
(0.05)\end{array}$ & $\begin{array}{c}0.61 \\
(0.02)\end{array}$ \\
\hline B Right & $\begin{array}{c}0 \cdot 30 \\
(0 \cdot 02)\end{array}$ & $\begin{array}{c}0.19 \\
(0.01)\end{array}$ & $\begin{array}{c}0.27 \\
(0.02)\end{array}$ & $\begin{array}{c}0.61 \\
(0.04)\end{array}$ & $\begin{array}{c}1.88 \\
(0.07)\end{array}$ & $\begin{array}{c}1.22 \\
(0.04)\end{array}$ & $\begin{array}{c}0.61 \\
(0.01)\end{array}$ \\
\hline$C$ Left & $\begin{array}{c}0.53 \\
(0.02)\end{array}$ & $\begin{array}{c}0.47 \\
(0.02)\end{array}$ & $\begin{array}{c}0.57 \\
(0.02)\end{array}$ & $\begin{array}{c}0.86 \\
(0.04)\end{array}$ & $\begin{array}{c}1 \cdot 15 \\
(0.04)\end{array}$ & $\begin{array}{c}0.94 \\
(0.04)\end{array}$ & $\begin{array}{c}0.61 \\
(0.02)\end{array}$ \\
\hline C Right & $\begin{array}{c}0.46 \\
(0.02)\end{array}$ & $\begin{array}{c}0.46 \\
(0.02)\end{array}$ & $\begin{array}{c}0.55 \\
(0.02)\end{array}$ & $\begin{array}{c}0.78 \\
(0.03)\end{array}$ & $\begin{array}{c}1.09 \\
(0.05)\end{array}$ & $\begin{array}{c}0.84 \\
(0.03)\end{array}$ & $\begin{array}{c}0.58 \\
(0.02)\end{array}$ \\
\hline D Left & $\begin{array}{c}0.44 \\
(0.02)\end{array}$ & $\begin{array}{c}0.32 \\
(0.02)\end{array}$ & $\begin{array}{c}0.43 \\
(0.03)\end{array}$ & $\begin{array}{c}0.72 \\
(0.05)\end{array}$ & $\begin{array}{c}1.50 \\
(0.07)\end{array}$ & $\begin{array}{c}1.07 \\
(0.04)\end{array}$ & $\begin{array}{c}0.64 \\
(0.03)\end{array}$ \\
\hline D Right & $\begin{array}{c}0.43 \\
(0.02)\end{array}$ & $\begin{array}{c}0.32 \\
(0.02)\end{array}$ & $\begin{array}{c}0.41 \\
(0.02)\end{array}$ & $\begin{array}{c}0.71 \\
(0.04)\end{array}$ & $\begin{array}{c}1.52 \\
(0.08)\end{array}$ & $\begin{array}{c}1 \cdot 10 \\
(0.04)\end{array}$ & $\begin{array}{c}0.63 \\
(0.02)\end{array}$ \\
\hline D Upper & $\begin{array}{c}0.44 \\
(0.02)\end{array}$ & $\begin{array}{c}0.35 \\
(0.02)\end{array}$ & $\begin{array}{c}0.44 \\
(0.03)\end{array}$ & $\begin{array}{c}0.73 \\
(0.05)\end{array}$ & $\begin{array}{c}1.47 \\
(0.07)\end{array}$ & $\begin{array}{c}1.07 \\
(0.04)\end{array}$ & $\begin{array}{c}0.65 \\
(0.02)\end{array}$ \\
\hline D Lower & $\begin{array}{c}0.42 \\
(0.02)\end{array}$ & $\begin{array}{c}0.30 \\
(0.02)\end{array}$ & $\begin{array}{c}0.39 \\
(0.03)\end{array}$ & $\begin{array}{c}0.71 \\
(0.05)\end{array}$ & $\begin{array}{c}1.54 \\
(0.08)\end{array}$ & $\begin{array}{c}1 \cdot 10 \\
(0.04)\end{array}$ & $\begin{array}{c}0.63 \\
(0.02)\end{array}$ \\
\hline
\end{tabular}

ABCD are the four patients. IB = Internal bronchiolar diameter; IA =Internal arterial diameter; EMA = External medial arterial diameter; and $\mathrm{EAA}=$ External adventitial arterial diameter. All values uncorrected for shrinkage during processing. Values in brackets are the standard errors of the mean.

Table 3 Results of statistical analysis of diameters and ratios for subject, left-right, method of inflation, slice, and upper and lower differences

\begin{tabular}{llllllll}
\hline & $I B$ & $I A$ & $E M A$ & $E A A$ & $I B / I A$ & $I B / E M A$ & $I B / E A A$ \\
\hline Patients & $\mathrm{P}<0.001$ & $\mathrm{P}<0.001$ & $\mathrm{P}<0.001$ & $\mathrm{P}<0.001$ & $\mathrm{P}<0.001$ & $\mathrm{P}<0.001$ & NS \\
Left-right & $\mathrm{P}<0.05$ & $\mathrm{P}<0.001$ & $\mathrm{P}<0.001$ & $\mathrm{P}<0.02$ & $\mathrm{P}<0.001$ & $\mathrm{P}<0.02$ & NS \\
Method of inflation & $\mathrm{NS}$ & $\mathrm{P}<0.001$ & $\mathrm{P}<0.001$ & $\mathrm{NS}$ & $\mathrm{P}<0.001$ & $\mathrm{P}<0.001$ & $\mathrm{NS}$ \\
Slice & $\mathrm{P}<0.01$ & $\mathrm{P}<0.001$ & $\mathrm{P}<0.001$ & $\mathrm{P}<0.001$ & $\mathrm{NS}$ & $\mathrm{NS}$ & $\mathrm{NS}$ \\
Upper-lower & $\mathrm{NS}$ & $\mathrm{NS}$ & $\mathrm{NS}$ & $\mathrm{NS}$ & $\mathrm{NS}$ & $\mathrm{NS}$ & NS \\
\hline
\end{tabular}

NS $=$ Not significant. 
statistical analysis of the various differences. There were statistically significant differences in the IB and EAA diameters between patients, right and left lungs, and slices but not between methods of inflation. For the IA and EMA diameters there were, in addition, significant differences between methods of inflation. The $\mathrm{IB} / \mathrm{IA}$ ratio varied significantly between methods of inflation but not between slices, the same pattern being observed in the IB/EMA ratio. For the IB/EAA ratio, however, no significant differences could be shown. The SE (expressed as percentage of the mean) for the IB/IA, IB/EMA, and IB/EAA ratios in each lung were $4.3 \% 3.5 \%$, and $2.8 \%$ respectively. There was no correlation between the IB diameter and the IB/EAA ratio indicating that the ratio is independent of the size of the bronchiole. There was a unimodal distribution of the IB/EAA ratios (fig 3).

The measured total inflated lung volume of the lungs of patient $\mathrm{D}$ was $3850 \mathrm{ml}$ compared with a predicted normal of $5100 \mathrm{ml}$ (predicted total lung capacity for height plus $14 \%$ to account for lung tissue).

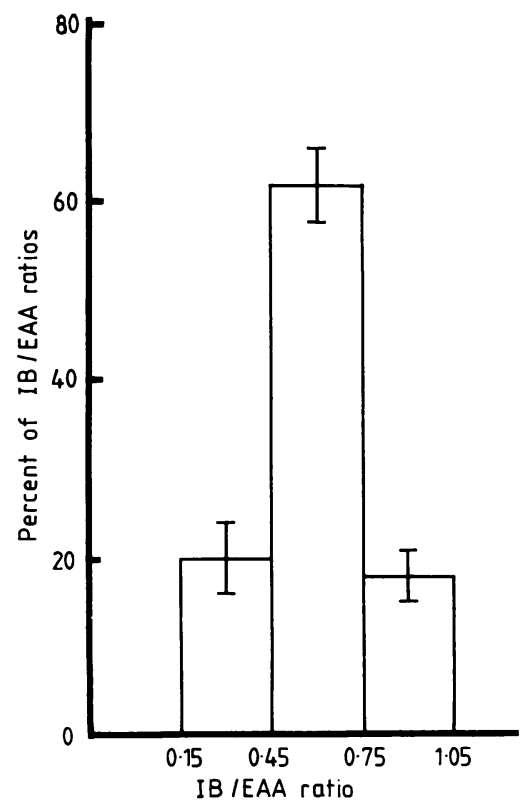

Fig 3 Percentage distribution of $I B / E A A$ ratios. $I B=$ Internal bronchiolar diameter, and $E A A=$ External adventitial arterial diameter.

\section{Discussion}

The branching pattern of the pulmonary artery is such that branches are given off to accompany the branches of the bronchial tree. In addition there are many supernumerary branches that do not accompany the bronchial tree (Elliott and Reid, 1965). Because of the close association between the bronchi and arteries in the lung it seemed attractive to attempt to use the arteries as a reference for bronchiolar calibre. It has been previously noted, however, that the internal diameters of the arterial branches do not diminish in a regular fashion with distance from the hilum (Elliott and Reid, 1965). It needed to be shown whether this made the arteries unsuitable as a reference.

There are several possible sources of error in our data. Firstly, individual lungs may have been over or under-inflated and, secondly, the methods used to measure diameters may possibly have led to errors. It is difficult to know if the lungs were fully inflated, but certainly there was absence of pleural wrinkling in all lungs. It has been shown previously that an inflation pressure of $25 \mathrm{~cm}$ of water may lead to over- or under-inflation of the lungs compared to predicted normal lung volume (Matsuba and Thurlbeck, 1972). Since small airway diameter probably varies with lung volume, variation in the degree of inflation between lungs would lead to variation in IB diameters, and this was indeed observed.

Another experimental error may contribute to the IB/IA and IB/EMA differences. The three arterial diameters were measured along the same line for obliquely sectioned vessels. Often, however, the lesser diameter for the three measurements did not all lie on exactly the same line, and some variability must have resulted from this. There may, in addition, be true variability in these ratios, unrelated to experimental methods. As previously mentioned, internal arterial diameters do not diminish in a regular manner with successive branching. Furthermore, age-related differences may be influenced by intimal and medial changes that have been documented by Semmens (1970) and Shelton et al (1977). Such differences may affect the EAA diameter much less than the IA or EMA diameters.

The IB/EAA ratio showed no variability between patients, right and left lungs, or between sites in the lung (slice and upper and lower halves). As the components of this ratio-that is, the IB and EAA diameters - did vary, this suggests that they varied in the same direction and by the same amount in each individual pair. It is not surprising that the IB/EAA ratio was independent of the method of inflation as neither the IB nor EAA diameters showed significant variation due to this factor. This indicates that the radial traction 
exerted on the arterial adventitia by the lungs expanded by bronchial inflation alone produces a similar EAA diameter to that achieved by added arterial inflation. The adventitia of pulmonary artery is derived from mesenchyme and consists of loose connective tissue in which spaces can often be seen (Spencer, 1968). Teleologically, the adventitia presumably serves to cushion the arteries from the lung, allowing blood to flow independently of respiratory movements (Spencer, 1968). For these reasons the IB/EAA ratio may be expected to be relatively independent of intimal and medial changes related to age, disease, or other factors and therefore give a true reflection of changes in bronchiolar calibre.

The many observations in this study were made to document fully the variability of the measurements and ratios, and this number of observations would not be suitable for routine use. Preliminary work in abnormal lungs, however, shows that an SE (percent of the mean) of $10 \%$ can be obtained from measurements of 30 pairs of bronchioles and arteries.

Our aim was to find a method for measuring objectively the luminal size of small airways so that abnormalities in disease can be recorded. Such a measurement, to be of use in routine assessment of lung pathology, would ideally be independent of the method and exact degree of inflation, the site within the lung, and the age of the patient. In this study the IB/EAA ratio best fulfilled these criteria. Certainly arterial inflation in addition to bronchial inflation is unnecessary, and the site in the lung from which the sections are taken is of no importance. We had too few lungs to study the effects of age, but the mean values for the old and young lungs were remarkably similar.

It seems, therefore, that the IB/EAA ratio is a good index of bronchiolar size, which could be used to measure narrowing of small airways. Further studies in diseased lungs should confirm or deny its usefulness.

We thank Dr R Newland, Mr A Smith, and the Department of Anatomical Pathology, Repatriation General Hospital, Concord, and $\mathrm{Mr}$ M Jones, Department of Medicine, University of Sydney.

\section{References}

Bignon, J, Andre-Bougaran, J, and Brouet, G (1970). Parenchymal, bronchiolar, and bronchial measurements in centrilobular emphysema. Relation to weight of right ventricle. Thorax, 25, 556-567.
Depierre, A, Bignon, J, Lebeau, A, and Brouet, G $\Rightarrow$ (1972). Quantitative study of parenchyma and small $\stackrel{9}{\rightarrow}$ conductive airways in chronic nonspecific lung 0 disease. Chest, 62, 699-708.

Elliott, F M, and Reid, L (1965). Some new facts about the pulmonary artery and its branching $\mathbb{D}$ pattern. Clinical Radiology, 16, 193-198.

Heard, B E (1958). A pathological study of emphysema \& of the lungs with chronic bronchitis. Thorax, 13, $\overrightarrow{0}$ 136-149.

Hogg, J C, Macklem, P T, and Thurlbeck, W M $\vec{\omega}$ (1968). Site and nature of airway obstruction in $\odot$ chronic obstructive lung disease. New England Jour- $\vec{x}$ nal of Medicine, 278, 1355-1360.

Linhartová, A, Anderson, A E, and Foraker, A G (1971). Radial traction and bronchiolar obstruction $\omega$ in pulmonary emphysema. Observed and theoretical of aspects. Archives of Pathology, 92, 384-391.

Linhartová, A, Anderson, A E, and Foraker, A G 을 (1973). Nonrespiratory bronchiolar deformities. Graphic assessment in normal and emphysematous lungs. Archives of Pathology, 95, 45-47.

Linhartová, A, Anderson, A E, and Foraker, A G (1977). Further observations on luminal deformity and stenosis of non-respiratory bronchioles in pulmonary emphysema. Thorax, 32, 53-59.

Macklem, P T, Thurlbeck, W M, and Fraser, R G (1971). Chronic obstructive disease of small airways. Annals of Internal Medicine, 74, 167-177.

Martin, C J, Cochran, T H, and Katsura, S (1968). Tuberculosis, emphysema and bronchitis. American Review of Respiratory Disease, 97, 1089-1093.

Martin, C J, Katsura, S, and Cochran, T H (1970). The relationship of chronic bronchitis to the diffuse obstructive pulmonary syndrome. American Review of Respiratory Disease, 102, 362-369.

Matsuba, K, and Thurlbeck, W M (1971). The number and dimensions of small airways in nonemphysematous lungs. American Review of Respiratory Disease, 104, 516-524.

Matsuba, K, and Thurlbeck, W M (1972). The number and dimensions of small airways in emphysematous lungs. American Journal of Pathology, 67, 265-275.

Semmens, M (1970). The pulmonary artery in the 0 normal aged lung. British Journal of Diseases of the Chest, 64, 65-72.

Shelton, D M, Keal, E, and Reid, L (1977). The pulmonary circulation in chronic bronchitis and emphy- $\mathcal{N}$ sema. Chest, 71, 303-306.

Spencer, H (1968). Pathology of the Lung. 2nd edn, p 45. Pergamon Press, Oxford.

Zamel, N, Hogg, J, and Gelb, A (1976). Mechanisms 으 of maximal expiratory flow limitation in clinically 0 unsuspected emphysema and obstruction of the peripheral airways. American Review of Respiratory Disease, 113, 337-345.

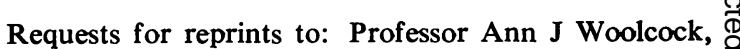
Department of Medicine, University of Sydney, Sydney, NSW 2006. 